\title{
Asymmetric Mizoroki-Heck Reactions: Generation of Quaternary Stereocenters and Cascade Cyclizations
}

\author{
Ane R. Azcargorta, ${ }^{1}$ Iratxe Barbolla, ${ }^{1}$ E. Coya, ${ }^{1}$ Nuria Sotomayor ${ }^{1}$, and Esther Lete ${ }^{1 *}$ \\ 1 Departamento de Química Orgánica II, Facultad de Ciencia y Tecnología, Universidad del País \\ Vasco / Euskal Herriko Unibertsitatea UPV/EHU. Apdo. 644. 48080 Bilbao, Spain. \\ http://www.ehu.es/oms \\ * Author to whom correspondence should be addressed; E-Mail: esther.lete@ehu.es; \\ Tel.: +34 946012576; Fax: +34 946012748.
}

\section{Published: 4 December 2015}

\begin{abstract}
Mizoroki-Heck reaction constitutes an effective method for the formation of quaternary stereocenters. This reaction has been applied to functionalized 2-alkenylpyrroles as substrates in which the $\beta$-elimination is blocked by a methyl substituent. 10,10-Disubstituted pyrrolo[1,2-b]isoquinolines can be obtained using different palladium catalysts and chiral bidentate phosphanes as ligands, although with low enantioselectivities. In some cases, competition between Mizoroki-Heck reaction and $\mathrm{C}-\mathrm{H}$ direct arylation reaction on the pyrrole nucleus has also been observed for this type of polyfunctionalized substrates. Finally, we have shown that quaternary stereocenters can be generated using chiral phosphane ligands as $(R)$ BINAP, through a cascade polyene cyclization. This procedure has been successfully applied to the construction of tetracyclic framework of Lycorine class of Amaryllidaceae alkaloids.
\end{abstract}

Keywords: Palladium; Heck reaction; cascade reactions; alkaloids; pyrrolo[1,2- $b]$ isoquinolines

\section{Introduction}

The Mizoroki-Heck reaction of aryl and vinyl halides with alkenes has developed into one of the most important carbon-carbon bond-forming reactions in organic synthesis. ${ }^{1}$ The intramolecular variant represents an extremely powerful method for the construction of small and medium-size rings. Particularly in recent years, this procedure has become an effective method for the formation of quaternary stereocenters, even in an asymmetric fashion. ${ }^{2}$ The use of substrates where the $\beta$-elimination is blocked by a substituent allows to maintain the $\mathrm{sp}^{3}$ centre formed after the migratory insertion, 
driving the elimination to another $\beta^{\prime}$ position, and generating a quaternary stereocenter.

In the context of our research program in palladium catalyzed reactions, ${ }^{3}$ we have previously reported that pyrrolo[1,2$b$ ]isoquinolines can be accessed via the intramolecular palladium-catalyzed Heck reaction of 2-alkenyl-substituted $N$-(oiodobenzyl)pyrroles, avoiding the direct arylation on the pyrrole nucleus by choosing the appropriate catalytic system. ${ }^{4}$ The procedure has also been applied to the selective synthesis of medium-sized rings and to (hetero)fused indolizine systems. ${ }^{5}$ Thus, our next goal was to examine the possibility of generating quaternary stereocenters on C-10 of the pyrroloisoquinoline skeleton. For that purpose, we selected 2alkenylpyrroles as substrates in which the $\beta$ elimination is blocked by a methyl substituent.

\section{Results and Discussion}

To start studying the generation of a quaternary center, we selected as substrate $N$-benzylpyrrole 1a (Scheme 1).

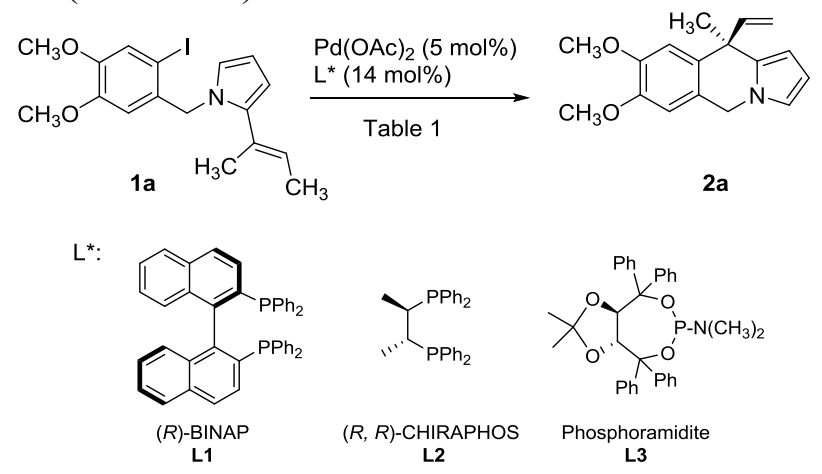

Scheme 1

The reaction was tested first in the racemic version. When 1a was treated with $\mathrm{Pd}(\mathrm{OAc})_{2}(5$ $\mathrm{mol} \%)$ in the presence of $\mathrm{PPh}_{3}(14 \mathrm{~mol} \%)$ in refluxing THF, racemic $\mathbf{2 a}$ was obtained, although in a low yield (37\%). The reaction conditions were optimized for the enantioselective version. Thus, three privileged ligands were selected: $(R)$-BINAP (L1), Chiraphos (L2) and phosphoramidite L3 (Scheme 1). As shown on Table 1, when the reaction was carried out using $(R)$-BINAP in THF, pyrroloisoquinoline $\mathbf{2 a}$ was obtained with a promising ee $(78 \%$ ee), although in a very low yield (entry 1). The use of PMP as a base resulted in an increased yield, though with loss of enantioselectivity (entry 2). The use of Chiraphos (L2) gave almost no conversion (entries 3, 4), while phosphoramidite $\mathbf{L} 3$ provided the opposite enantiomer, but with low yields and enantioselectivities. The change of the solvent to DMF did not improve the results.

. Table 1. Cyclization reactions of $\mathbf{1 a}$

\begin{tabular}{cccccc} 
Entry & Base & Solvent & $\mathbf{L}^{*}$ & $\begin{array}{c}\text { 2a } \\
\text { Yield (\%) }\end{array}$ & $\begin{array}{l}\boldsymbol{e e} \\
(\%)^{[\mathrm{a}]}\end{array}$ \\
\hline 1 & - & $\mathrm{THF}^{[\mathrm{b}]}$ & $\mathbf{L 1}$ & 5 & 78 \\
2 & $\mathrm{PMP}$ & $\mathrm{THF}^{[\mathrm{b}]}$ & $\mathbf{L 1}$ & 12 & 47 \\
3 & - & $\mathrm{THF}^{[\mathrm{b}]}$ & $\mathbf{L 2}$ & 8 & 2 \\
4 & $\mathrm{PMP}$ & $\mathrm{THF}^{[\mathrm{b}]}$ & $\mathbf{L 2}$ & - & - \\
5 & - & $\mathrm{THF}^{[\mathrm{b}]}$ & $\mathbf{L 3}$ & 12 & -25 \\
6 & $\mathrm{PMP}$ & $\mathrm{THF}^{[\mathrm{b}]}$ & $\mathbf{L 3}$ & 16 & -26 \\
7 & - & $\mathrm{DMF}^{[\mathrm{c}]}$ & $\mathbf{L 1}$ & 47 & 18 \\
8 & - & $\mathrm{DMF}^{[\mathrm{c}]}$ & $\mathbf{L 2}$ & 5 & 22 \\
9 & $\mathrm{PMP}$ & $\mathrm{DMF}^{[\mathrm{c}]}$ & $\mathbf{L 3}$ & 24 & 8 \\
\hline
\end{tabular}

${ }^{[a]}$ Determined by Chiral Stationary Phase HPLC (Chiralcel OZ3 2\% hexane/i-PrOH). ${ }^{[\mathrm{b}]}$ Reflux ${ }^{[\mathrm{c}]} 80^{\circ} \mathrm{C}$. 

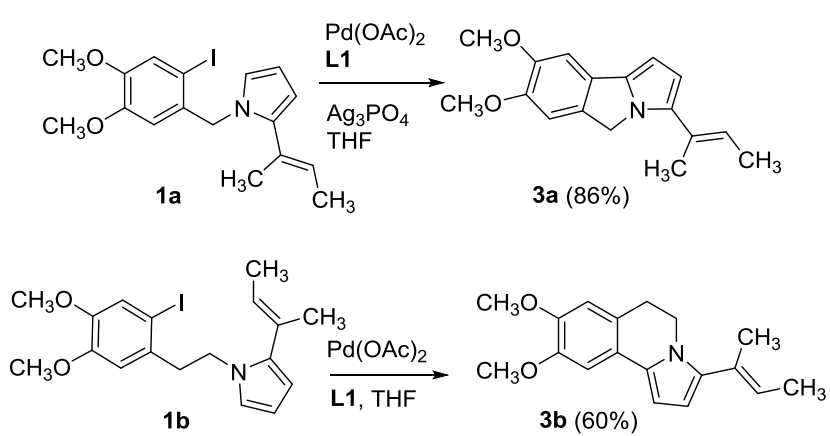

$1 b$
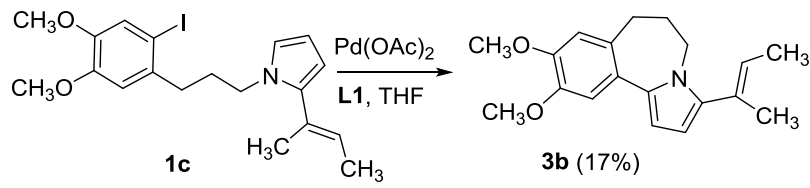

Scheme 2

On the other hand, when silver salts were added to try to improve the enantioselectivity, 1a afforded the pyrroloisoindol 3a, through a direct $\mathrm{C}-\mathrm{H}$ arylation reaction (Scheme 2). In a similar fashion, it was observed that the direct $\mathrm{C}-\mathrm{H}$ arylation reaction predominates under all conditions tested when $\mathbf{1 b}$ and $\mathbf{1 c}$ were treated with $\mathrm{Pd}(\mathrm{OAc})_{2}$, obtaining the pyrroloisoquinoline $\mathbf{3 b}$ and pyrrolobenzazepine $\mathbf{3} \mathbf{c}$, not observing the formation of the Heck products, as shown in Scheme 2.

In view of these results, we decided to introduce a protected allylic alcohol moiety in the alkene, to favor the cyclization, as a result of the formation of an enol ether. Thus, when 1d was treated with $\mathrm{Pd}\left(\mathrm{PPh}_{3}\right)_{4}$ in toluene, an excellent yield of the pyrroloisoquinoline $\mathbf{2 d}$ was obtained, as a mixture of $Z / E$ isomers (Scheme 3 ), forming a quaternary centre. The enol ether could be deprotected and the resulting aldehyde was reduced to afford the 10-hydroxymethyl derivative 4 in high yield. Unfortunately, when the cyclization was attempted using $(R)$-BINAP in different solvents, the yields of $\mathbf{2 d}$ were moderate (38 to $65 \%$ ) and the enantioselectivities were very poor (up to $11 \% e e$ ).
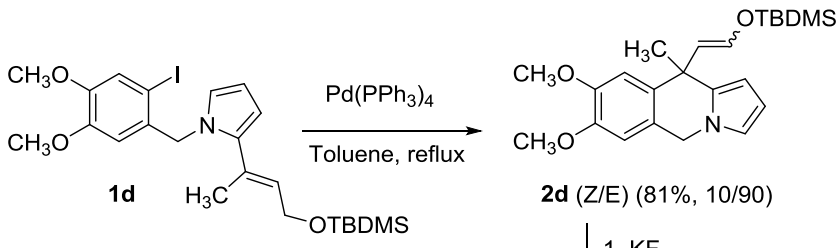

2d (Z/E) $(81 \%, 10 / 90)$

1. KF, $\mathrm{MeOH} / \mathrm{THF}$ 2. $\mathrm{NaBH}_{4}$<smiles>COc1cc2c(cc1OC)C(C)(CCO)c1cccn1C2</smiles>

\section{Scheme 3}

Finally, we reasoned that the formation of the quaternary center would be favored if the alkylpalladium is involved in a second reaction, in a cascade process Thus, we have recently shown that that quaternary stereocenters can be generated using chiral phosphane ligands as $(R)$ BINAP (L1), through a cascade polyene cyclization. $^{6}$ This procedure has been successfully applied to the construction of tetracyclic framework of Lycorine class of Amaryllidaceae alkaloids. ${ }^{7}$ Thus, $N$-benzyl 2,3dialkenyl pyrroles 5 undergo sequential 6-exo/6endo cyclizations to yield enantiomerically enriched $(11 \mathrm{~b} R)$-substituted pyrrolophenanthridines. $(R)$-BINAP (L1) has been shown to be the most efficient chiral phosphane ligand. The reaction can be extended to various substitution patterns on the aromatic ring, and also to heteroaromatic rings.
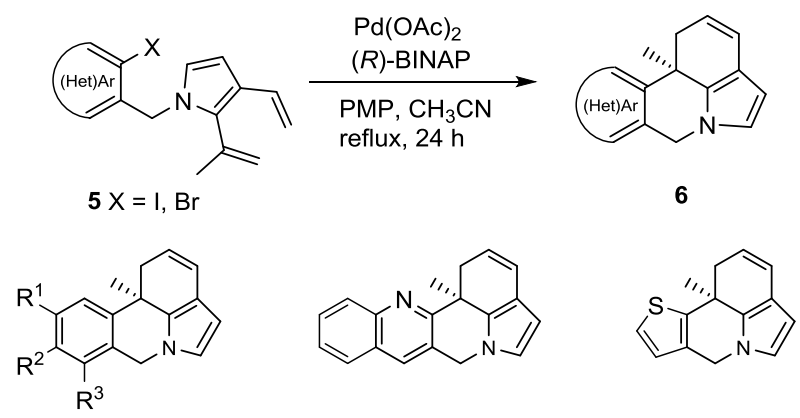

$R^{1}, R^{2}, R^{3}=H, O_{3}, O B n, F, \ldots$

$50-72 \%, 60-72 \%$ ee

$11 \%, 99 \%$ ee

$62 \%, 68 \%$ ee 


\section{Conclusions}

Mizoroki-Heck reaction on 2-substituted $\mathrm{N}$ benzylpyrroles in which the $\beta$-elimination is blocked by a substituent allows the generation of a quaternary stereocenter on C-10 position of the pyrrolo[1,2-b]isoquinoline skeleton. However, the reaction proceeds only with moderate yield and low enantioselectivity when chiral phosphanes (L1-L3) are used as ligands. The generation of larger rings is not possible, as the $\mathrm{C}-\mathrm{H}$ direct arylation reaction predominates under all conditions tested. The generation of the quaternary stereocenter is favored when a protected allylic alcohol moiety is used obtaining high yields of the C-10 disubstituted pyrroloisoquinoline, but the reaction was not efficient when chiral phosphanes were used. Finally, the formation of the quaternary stereocenter is more efficient when the resulting alkylpalladium intermediate is involved in a cascade process. Thus, 2,3-dialkenyl pyrroles undergo sequential 6-exo/6-endo cyclizations to afford $(11 \mathrm{~b} R)$-substituted pyrrolophenanthridines in good yields and enantioselectivities. This procedure allows a rapid and efficient access to a wide variety of enantiomerically enriched $\mathrm{C}-11 \mathrm{~b}$ substituted lycorane analogues.

\section{Acknowledgments}

Ministerio de Economía y Competitividad (CTQ2013-41229-P), Gobierno Vasco (IT-62313) and Universidad del País Vasco / Euskal Herriko Unibertsitatea UPV/EHU (UFI11/22, PPM12/03) are gratefully acknowledged for their

financial support. We also thank Gobierno Vasco for grants (ARA, EC). Technical and human support provided by Servicios Generales de Investigación SGIker (UPV/EHU, MINECO, GV/EJ, ERDF and ESF) is also acknowledged.

\section{Conflicts of Interest}

The authors declare no conflict of interest.

\section{References and Notes}

1. a) Oestreich M. Ed, The Mizoroki-Heck reaction, Wiley: Chichester, 2009; b) M. Larhed, Ed., Science of Synthesis - Cross Coupling and Heck-Type Reactions, Vol. 3, Thieme: Stuttgart, 2013.

2. a) Tietze, L. F.; Ila, H; Bell, H. P. Chem. Rev. 2004, 104, 3453-3516. b) McCartney, D.; Guiry, P. J. Chem. Soc. Rev. 2011, 40, 5122-5150.

3. a) Martínez-Estíbalez, U.; García-Calvo, O.; Ortiz-de-Elguea, V.; Sotomayor, N.; Lete E.; Eur. J. Org. Chem. 2013, 3013-3022;; b) Ortiz-de-Elguea, V.; Sotomayor, N.; Lete, E. Adv. Synth. Catal. 2015, 356, 463-473

4. Lage, S.; Martínez-Estibalez, U.; Sotomayor, N.; Lete, E. Adv. Synth. Catal. 2009, 351, 24602468

5. Coya, E.; Sotomayor, N.; Lete E. Adv. Synth. Catal. 2014, 356, 1853-1865

6. Coya, E.; Sotomayor, N.; Lete E. Adv. Synth. Catal. 2015, 357, 3206-3214. 
http://sciforum.net/conference/mol2net-1

7. Jin, Z.; Nat. Prod. Rep. 2013, 30, 849-868, and previous reports on these series.

(C) 2015 by the authors; licensee MDPI, Basel, Switzerland. This article is an open access article distributed under the terms and conditions defined by MDPI AG, the publisher of the Sciforum.net platform. Sciforum papers authors the copyright to their scholarly works. Hence, by submitting a paper to this conference, you retain the copyright, but you grant MDPI AG the non-exclusive and unrevocable license right to publish this paper online on the Sciforum.net platform. This means you can easily submit your paper to any scientific journal at a later stage and transfer the copyright to its publisher (if required by that publisher). (http://sciforum.net/about ). 